\title{
Pregnancy Outcome in Women With Familial Mediterranean Fever: A Retrospective Analysis of 50 Cases With a 10-Year Experience
}

\author{
Aslıhan YAZICIOĞLU, Mert TURGAL, Özge Senem YÜCEL, Özgür ÖZYÜNCÜ, M. Sinan BEKSAÇ \\ Department of Obstetrics and Gynecology, Medical Faculty of Hacettepe University, Ankara, Turkey
}

\begin{abstract}
Objectives: Since little is known about perinatal outcome of patients with familial Mediterranean fever (FMF), we report our clinical experience about pregnant women with FMF in this study.

Patients and methods: Fifty pregnant women (mean age 29.5 years; range 19 to 39 years) with FMF were enrolled between January 2002 and December 2012. Patient data were collected from computerized database. Clinical characteristics including demographics, pregnancy outcome, maternal and fetal complications during pregnancy or delivery, hypercoagulation disorders and colchicine use were recorded. Labor patterns and perinatal outcomes were assessed.

Results: The mean gestational week at delivery was 36.3. Maternal complications were encountered in two patients including hemodialysis associated sepsis and cerebrovascular accident. We did not encounter FMF attacks in the course of pregnancies. A total of 42 patients (84\%) were on drug therapy of colchicine. Two congenital anomalies were encountered, one was tanatrophic dysplasia (on colchicine therapy) and the other was an open type neural tube defect (not on colchicine therapy). Patients were also searched for thrombophilias and 15 (30\%) had positive test result. The course of the pregnancy was free of venous complications with low molecular weight heparin prophylaxis. Preterm delivery, low birth weight and congenital anomalies were not significantly associated with colchicine therapy.

Conclusion: A favorable pregnancy and perinatal outcome may be expected in patients with FMF treated with colchicine. However, close followup with prenatal test and laboratory investigations for maternal and fetal well-being including hypercoagulation disorders are thought to be essential.

Key words: Colchicine; Familial Mediterranean fever; perinatal outcome; pregnancy outcome; thrombophilia.
\end{abstract}

Familial Mediterranean fever (FMF) or recurrent polyserositis is a recessively inherited disorder, clinically characterized by inflammation of the serosal, synovial and cutaneous tissues with clinical fever episodes which usually limits itself. ${ }^{1}$ Mutations in the Mediterranean fever (MEFV) gene on the short arm of chromosome 16 are the most probable cause. ${ }^{2}$ The disease mostly affects Turks, Armenians, Arabs and non-Ashkenazi Jews. ${ }^{3}$ However, due to emigration of those people from the Mediterranean basin throughout history, FMF cases may be encountered all over the world, mainly in Western Europe. ${ }^{1}$ The most catastrophic complication of FMF is amyloidosis, which may lead to end stage renal disease and eventually death, and may also involve other organs and tissues. Colchicine is the drug of choice for FMF, used to prevent and decrease the severity of FMF attacks and the development of amyloidosis. ${ }^{1}$ Colchicine use during pregnancy and lactation is reported to be safe. ${ }^{2}$ In a gynecological perspective, acute peritonitis attacks during pregnancy may lead to eventual abortion and preterm delivery. The course and outcome of pregnancies treated with colchicine was comparable with that of the general population. ${ }^{4}$ Furthermore, little is known about the perinatal outcome of patients with FMF. Herein, we report our clinical experience in pregnant women with FMF. The article describes 
epidemiologic data, the course of pregnancies and maternal and perinatal outcomes of pregnant women with FMF.

\section{PATIENTS AND METHODS}

Fifty pregnant women with FMF who were admitted to the Department of Obstetrics and Gynecology, Materno-Fetal Unit in Hacettepe University Hospital, Ankara, Turkey were enrolled in the study between June 2002 and July 2012. Informed consents were obtained from all patients. All patients were clinically diagnosed with FMF according to the Tel Hashomer criteria. ${ }^{5}$ Patient data were collected from a computerized database that consists of information recorded during antenatal visits and just after delivery by the same obstetrician. During the time of the study, the pregnancies were followed according to the standard antenatal follow-up protocol of high-risk pregnancies. Antenatal visits were scheduled every four weeks for the first 20 weeks and then every two weeks until 36 weeks and then weekly until delivery. During antenatal visits, routine obstetric ultrasound and laboratory investigations were performed for all pregnant women. All patients were also screened for hypercoagulation disorders, and patients who tested positive were put on prophylactic doses of low molecular weight heparin during the course of their pregnancies. First trimester ultrasound scans for nuchal translucency and first trimester biochemical markers were assessed, while second trimester ultrasound anomaly scan was routinely performed at 20 weeks' gestation for all pregnancies. Clinical characteristics including maternal age, parity, pregnancy outcome, maternal and fetal complications during pregnancy or delivery and colchicine usage were evaluated. Labor characteristics and perinatal outcomes including gestational age, Apgar scores, congenital anomalies, birth weight, perinatal mortality and neonatal complications were assessed.

Statistical analysis was performed with the SPSS for Windows version 17.0 software program (SPSS Inc., Chicago, IL, USA). The relationship between the use of colchicine and perinatal outcomes was evaluated with the independentsamples $\mathrm{T}$ test.

\section{RESULTS}

Demographic characteristics of the study group are shown in Table 1. Three patients had renal amyloidosis, one had end stage renal disease and one had polycystic kidney disease. All patients were evaluated for thrombophilias, those with positive test results for hypercoagulation disorders, were put on low-molecular weight heparin prophylaxis during the course of their pregnancies. We observed no thromboembolic attack. Gestational hypertensive diseases were

\begin{tabular}{|lcccr|}
\hline \multicolumn{5}{|l}{ Table 1. Demographic characteristics of the study group } \\
\hline Demographic characteristics & $\mathrm{n}$ & $\%$ & Mean \pm SD & Range \\
\hline Mean maternal age (week) & & & $29.5 \pm 5.1$ & $19-39$ \\
Parity & 33 & 66 & & \\
$\quad$ Primiparous & 17 & 34 & & \\
$\quad$ Multiparous & & & & \\
Type of delivery & 31 & 62 & \\
$\quad$ Cesarean delivery & 19 & 38 & \\
$\quad$ Vaginal delivery & 42 & 84 & & \\
Colchicine usage & 8 & 16 & \\
$\quad$ Present & 15 & 30 & \\
$\quad$ Absent & 35 & 70 & \\
Hypercoagulation disorders & 4 & 26.70 & \\
$\quad+$ & 2 & 13.35 & \\
$\quad$ Factor V leiden gene mutation & 3 & 20.00 & \\
Low levels of protein C activity & 2 & 13.35 & \\
PAI-1 4G/5G polymorphism & 4 & 26.70 & \\
Prothrombin G20210A & & & \\
Antiphospholipid syndrome & &
\end{tabular}




\begin{tabular}{|c|c|c|c|c|}
\hline Perinatal characteristics & $\mathrm{n}$ & $\%$ & Mean \pm SD & Range \\
\hline Mean gestational age at delivery (week) & & & $36.3 \pm 3.8$ & $24-40$ \\
\hline Term (>37 week) & 38 & 76 & & \\
\hline Preterm (<37 week) & 12 & 24 & & \\
\hline Mean birth weight (gr) & & & $2841.6 \pm 839.2$ & $430-4190$ \\
\hline Normal birth weight (2500-4000 gr) & 38 & 74 & & \\
\hline Low birth weight $(<2500 \mathrm{gr})$ & 11 & 22 & & \\
\hline Macrosomia (>4000 gr) & 2 & 4 & & \\
\hline \multicolumn{5}{|l|}{ Number of gestation } \\
\hline Singleton & 49 & 98 & & \\
\hline Multiple gestation & 1 & 2 & & \\
\hline Live birth & 47 & 94 & & \\
\hline Stillbirth & 1 & 2 & & \\
\hline Neonatal death & 3 & 6 & & \\
\hline \multicolumn{5}{|l|}{ Apgar scores (at 1 and 5 minutes) } \\
\hline$<7$ & 3 & 6 & & \\
\hline$>7$ at 1 and 5 minutes & 48 & 94 & & \\
\hline \multicolumn{5}{|l|}{ Congenital anomalies } \\
\hline Present & 2 & 4 & & \\
\hline Absent & 49 & 96 & & \\
\hline \multicolumn{5}{|l|}{ Admission to NICU } \\
\hline Present & 13 & 26 & & \\
\hline Absent & 38 & 74 & & \\
\hline Mean length of stay in NICU (days) & & & $12.4 \pm 10.6$ & $1-69$ \\
\hline \multicolumn{5}{|l|}{ Neonatal complications } \\
\hline Necrotising enterocolitis & 2 & 4 & & \\
\hline Respiratory distress syndrome & 6 & 12 & & \\
\hline Intracranial hemorrhage & 2 & 4 & & \\
\hline
\end{tabular}

also encountered; three cases of preeclampsia, one case of HELLP syndrome and two cases of gestational hypertension were observed. Maternal complications were encountered in two patients including hemodialysis associated with sepsis and cerebrovascular accident. We did not encounter FMF attacks in the course of pregnancies. Perinatal characteristics are given in Table 2. Preterm deliveries were induced due to fetal and maternal indications. Two congenital anomalies were encountered, one of which was thanatophoric dysplasia (on colchicine therapy) and the other an open type neural tube defect (not on colchicine therapy).
The independent-samples $\mathrm{T}$ test was used to assess the association between birth weight, gestational week at delivery and congenital anomalies and colchicine use. Preterm delivery $(p=0.058)$, low birth weight $(p=0.416)$ and congenital anomalies $(p=0.451)$ were not significantly associated with colchicine therapy. We observed a slight but statistically insignificant difference in gestational week at delivery between the colchicine groups (on therapy or not) with a $p$ value of 0.058 . This was thought to be due to the small number of the study group. The statistical details are shown in Table 3.

Table 3. The relationships between colchicine use and birth weight, gestational week at delivery and congenital anomalies

\begin{tabular}{|c|c|c|c|c|c|c|c|c|c|}
\hline & \multirow{2}{*}{$\begin{array}{l}\text { Birth weight (gr) } \\
\text { Mean } \pm \text { SD }\end{array}$} & \multirow[t]{2}{*}{$95 \% \mathrm{CI}$} & \multirow[t]{2}{*}{$p$} & \multirow{2}{*}{$\begin{array}{c}\begin{array}{c}\text { Gestational week } \\
\text { at delivery }\end{array} \\
\text { Mean } \pm \text { SD }\end{array}$} & \multirow[t]{2}{*}{$95 \% \mathrm{CI}$} & \multirow[t]{2}{*}{$p$} & \multicolumn{2}{|c|}{$\begin{array}{l}\text { Congenital } \\
\text { anomaly }\end{array}$} & \multirow[t]{2}{*}{$p$} \\
\hline & & & & & & & $\mathrm{n}$ & $\%$ & \\
\hline $\begin{array}{l}\text { Colchicine }(+)(\mathrm{n}=42) \\
\text { Colchicine }(-) \quad(\mathrm{n}=8)\end{array}$ & $\begin{array}{l}2809.8 \pm 885.1 \\
3008.8 \pm 551.2\end{array}$ & $706.33-308.35$ & 0.416 & $\begin{array}{l}36.1 \pm 4.0 \\
37.8 \pm 1.7\end{array}$ & $3.46-0.06$ & 0.058 & $\begin{array}{l}1.0 \\
1.0\end{array}$ & $\begin{array}{c}2.4 \\
12.5\end{array}$ & 0.451 \\
\hline
\end{tabular}




\section{DISCUSSION}

Familial Mediterranean fever is an inherited systemic disorder of inappropriate inflammation. This severe inflammatory response may result in increased levels of serum amyloid A, which may affect most of the internal organs including the kidneys in particular. ${ }^{2}$ Treatment of acute exacerbations and prevention of amyloidosis rely on colchicine therapy. Since colchicine use during pregnancy and lactation is shown to be safe, discontinuation of treatment is not recommended. In our study population, the courses of pregnancies were variable, all were attack-free but one had cerebrovascular accident as a result of FMF-related vasculitis and the other needed daily hemodialysis (and sepsis) due amyloidosis-related end stage renal disease. Both pregnancies ended up with induced preterm labor. We did observe perinatal morbidity or mortality in these patients. However, in our study population the course of pregnancies and perinatal outcomes were comparable with that in the study performed by Nabil et al. ${ }^{2}$

Conception in patients with renal amyloidosis is still a dilemma. In the medical literature, it is reported that renal amyloidosis may result in poor obstetric outcome. ${ }^{2}$ On the other hand, successful pregnancy outcomes with renal amyloidosis were also reported. ${ }^{6,7}$ In our study, we encountered three cases of renal amyloidosis, one out three had deterioration in renal function during pregnancy, however, the other two reported well. In this perspective, with close follow-up and consultation on maternal and perinatal morbidity and mortality to the couple, conception may be advisable.

An increased baseline inflammation independently from clinical remission has been reported in patients with $\mathrm{FMF}^{8}$ It has also been reported that a subclinical inflammation may exaggerate natural anticoagulant response. ${ }^{8}$ In case of inherited and acquired thrombophilias, the expected increase in anticoagulant response may become insufficient. The resultant hypercoagulable state of FMF would increase exponentially. Aksu et al. ${ }^{8}$ studied 27 attack-free non-pregnant FMF patients with 26 controls, anticoagulant-procoagulant balance with related laboratory parameters. They detected a hypercoagulable state in the FMF group, suggesting a subclinical inflammation. ${ }^{8}$ When combined with a thrombophylic situation, increased susceptibility to thrombosis in pregnancy would further worsen the clinical picture. Under such conditions, we recommend administration of low molecular weight heparin at prophylactic dosage during pregnancy. In another study evaluating the association between plasminogen activator inhibitor-1 (PAI-1) 4G/5G polymorphism and the severity of FMF, data suggested a protective role for the $4 \mathrm{G}$ allele while the $5 \mathrm{G}$ allele seemed to have a more severe clinical course for FMF. ${ }^{9}$ Due to our small study population and the small number of studies in the literature on this subject, further studies would be required to reach a definitive conclusion.

Neonatal outcome in our study was not different from the outcome in the study performed by Nabil et al. ${ }^{2}$ In medical literature there is no clear association between FMF or colchicine use and developmental defects. ${ }^{2}$ Diav-Citrin et al. ${ }^{10}$ suggested that pregnant patients with FMF who conceived while receiving colchicine had uneventful pregnancy and neonatal outcome. Our current approach is to recommend continuous colchicine before conception and during pregnancy and if feasible, to perform a first trimester combined screening. A study of 238 pregnancies in 116 women treated with colchicine before or during pregnancy did not show an increased frequency of fetal defects. ${ }^{2}$ This suggestion is consistent with our study results.

In conclusion, although our study population is small, favorable pregnancy and perinatal outcomes can be expected in patients with FMF treated with colchicine. In order to achieve a final conclusion, more comprehensive studies consisting of larger study groups are needed. Due to the subclinical inflammation leading to a hypercoagulable state, we recommend searching for thrombophilia and giving appropriate anticoagulant prophylaxis during the course of pregnancy. Furthermore, close follow-up with prenatal test and laboratory investigations for maternal and fetal well-being are suggested to be essential.

\section{Declaration of conflicting interests}

The authors declared no conflicts of interest with respect to the authorship and/or publication of this article. 


\section{Funding}

The authors received no financial support for the research and/or authorship of this article.

\section{REFERENCES}

1. Mijatovic V, Hompes PG, Wouters MG. Familial Mediterranean fever and its implications for fertility and pregnancy. Eur $\mathrm{J}$ Obstet Gynecol Reprod Biol 2003;108:171-6.

2. Nabil H, Zayed A, State O, Badawy A. Pregnancy outcome in women with familial Mediterranean fever. J Obstet Gynaecol 2012;32:756-9.

3. Ben-Chetrit E, Ben-Chetrit A, Berkun Y, BenChetrit E. Pregnancy outcomes in women with familial Mediterranean fever receiving colchicine: is amniocentesis justified? Arthritis Care Res (Hoboken) 2010;62:143-8.

4. Ofir D, Levy A, Wiznitzer A, Mazor M, Sheiner E. Familial Mediterranean fever during pregnancy: an independent risk factor for preterm delivery. Eur $\mathrm{J}$ Obstet Gynecol Reprod Biol 2008;141:115-8.
5. Livneh A, Langevitz P, Zemer D, Zaks N, Kees $\mathrm{S}$, Lidar $\mathrm{T}$, et al. Criteria for the diagnosis of familial Mediterranean fever. Arthritis Rheum 1997;40:1879-85.

6. Ditkoff EC, Sauer MV. Successful pregnancy in a familial Mediterranean fever patient following assisted reproduction. J Assist Reprod Genet 1996;13:684-5.

7. Shimoni Y, Shalev E. Pregnancy and complicated familial Mediterranean fever. Int $J$ Gynaecol Obstet 1990;33:165-9.

8. Aksu G, Ozturk C, Kavakli K, Genel F, Kutukculer N. Hypercoagulability: interaction between inflammation and coagulation in familial Mediterranean fever. Clin Rheumatol 2007;26:366-70.

9. Bonyadi M, Shaghaghi Z, Haghi M, Dastgiri S. Plasminogen activator inhibitor-1 gene polymorphism in Iranian Azeri Turkish patients with FMF disease and its association with amyloidosis. Eur J Pediatr 2013;172:91-8.

10. Diav-Citrin O, Shechtman S, Schwartz V, Avgil-Tsadok M, Finkel-Pekarsky V, Wajnberg R, et al. Pregnancy outcome after in utero exposure to colchicine. Am J Obstet Gynecol 2010;203:144.e1-6. 